\title{
Microwave-assisted three-component reaction in conventional solvents and ionic liquids for the synthesis of amino-substituted imidazo[1,2-a]pyridines
}

\author{
Fadime Mert-Balci, Jürgen Conrad, and Uwe Beifuss* \\ Bioorganische Chemie, Institut für Chemie, Universität Hohenheim, Garbenstr. 30, 70599 \\ Stuttgart, Germany \\ E-mail: ubeifuss@uni-hohenheim.de
}

Dedicated to Professor Rainer Beckert on the occasion of his $60^{\text {th }}$ birthday

\begin{abstract}
3-Amino-substituted imidazo[1,2-a]pyridines can be prepared with yields up to $98 \%$ within a few minutes by microwave-assisted three-component reaction between 2-aminopyridines, aldehydes and isocyanides using montmorillonite as the catalyst and toluene as the solvent. The organic solvent can be replaced by ionic liquids. With guanidinium salts the microwave-assisted reaction can be performed in the absence of any further catalyst.
\end{abstract}

Keywords: Multicomponent reactions, imidazo[1,2-a]pyridines, microwaves, ionic liquids, imidazolium salts, guanidinium salts

\section{Introduction}

Recently, the application of microwaves in organic synthesis has become very popular. Microwave-assisted syntheses are a particularly attractive alternative to syntheses under thermal conditions since they often proceed much faster and deliver products with higher yields and higher purity. ${ }^{1}$ Upon conventional heating using an external heat source like an oil bath the energy transfer depends on the thermal conductivity of the sample to be penetrated, which is relatively slow and inefficient. In contrast, the energy of the microwaves is directly transferred to the molecules of the reaction mixture via dielectric heating. The heating is largely caused by dipolar polarization and ionic conduction.

Currently, the use of ionic liquids (ILs) as solvents and catalysts in organic transformations is receiving a great deal of interest. ${ }^{2}$ ILs have an ionic structure and they consist of an organic cation and an inorganic or organic anion. They have nearly no vapor pressure, are thermally and chemically robust, are non explosive, are convenient to use and can be recycled. Therefore, ILs 
can offer a more sustainable alternative to traditional organic solvents. In a number of reactions performed in ILs both a greatly increased reaction rate and a change in selectivities has been observed. These effects are probably due to polar interactions between the ILs and the substrates. Meanwhile, numerous organic transformations have been performed in ILs, ${ }^{2}$ and many of them from the field of heterocyclic chemistry. ${ }^{3}$ The significance which ILs have gained in organic synthesis is not only due to their solvent properties but to their catalytic effects as well. ${ }^{4}$ Due to their high polarity and high dielectricity constant ILs can be efficiently heated up by microwave irradiation. ${ }^{5}$ This is why reactions in ILs can benefit tremendously from microwave conditions. The best known ILs are the imidazolium salts which have been successfully used as solvents and/or catalysts in numerous chemical transformations. In addition, there is a large number of other ILs including pyridinium, phosphonium, ammonium and guanidinium salts.

Guanidinium salts, which are easily available by a number of methods, so far have been used in a small number of organic reactions, ${ }^{6}$ including aldol reactions, ${ }^{6, c}$ Heck reactions, ${ }^{6 b}$ and the oxidation of benzylic alcohols. ${ }^{6 e}$ Also, guanidinium salts have several properties predisposing them for applications as potential electrolytes in electrochemical devices such as lithium batteries, super capacitors, fuel cells and dye-sensitized solar cells. ${ }^{7}$

Multicomponent reactions are of great importance for the efficient assembly of compound libraries. ${ }^{8}$ The Groebke reaction is a particularly valuable three component reaction as it allows the synthesis of a number of relevant heterocyclic systems. It makes use of the reaction of 2aminoazines, aldehydes and isocyanides for the preparation of fused 3-aminoimidazoles, including imidazo[1,2- $a]$ pyridines, imidazo[1,2- $a]$ pyrimidines and imidazo[1,2- $a]$ pyrazines. Due to the marked biological activity of many compounds with an imidazo[1,2-a]pyridine skeleton ${ }^{9} \mathrm{a}$ number of different protocols for the Groebke reaction have been developed. ${ }^{10}$ The synthesis of imidazo[1,2-a]pyridines has been achieved in the presence of Brønsted acids ${ }^{10 \mathrm{~h}, \mathrm{i}}$ as well as with Lewis acids. ${ }^{10 b, d, g}$ The reaction can also be performed in the absence of any catalyst. ${ }^{10 a}$ The synthesis has also been performed under microwave conditions in the presence of montmorillonite ${ }^{10 \mathrm{f}}$ or $\mathrm{ZnCl}_{2}{ }^{10 \mathrm{~b}}$ There is also a report on the use of $[\mathrm{bmim}] \mathrm{Br}$ at room temperature to bring about the synthesis of imidazo[1,2-a]pyridines. ${ }^{10 \mathrm{c}}$ However, many of these methods suffer from different drawbacks such as long reaction times and/or the application of expensive catalysts. In order to establish a quick and efficient approach to this class of compounds we decided to study the influence of solvents on the microwave-assisted reaction between 2-aminopyridines, aldehydes and isocyanides in the presence of montmorillonite as the catalyst. It was planned to perform a model reaction in the absence and presence of solvents, including traditional organic solvents as well as ILs. Of particular interest was the use of guanidinium salts as reaction medium. 


\section{Results and Discussion}

Here we present our results on the microwave-assisted three-component synthesis of 3-amino substituted imidazo[1,2-a]pyridines 4 with montmorillonite as the catalyst under a variety of reaction conditions. The reaction between 2-aminopyridine (1a), benzaldehyde (2a) and benzylisocyanide (3a) was used as a model reaction. After some preliminary experiments it was found that the reaction could be run in the absence of any solvent when equimolar amounts of 1a, 2a and 3a were reacted in the presence of montmorillonite in a sealed vial under microwave conditions using a focused single mode microwave reactor for $5 \mathrm{~min}$ at $140^{\circ} \mathrm{C}$. Under these conditions $N$-benzyl-2-phenylimidazo[1,2-a]pyridin-3-amine (4a) could be isolated in 74\% yield (Scheme 1).

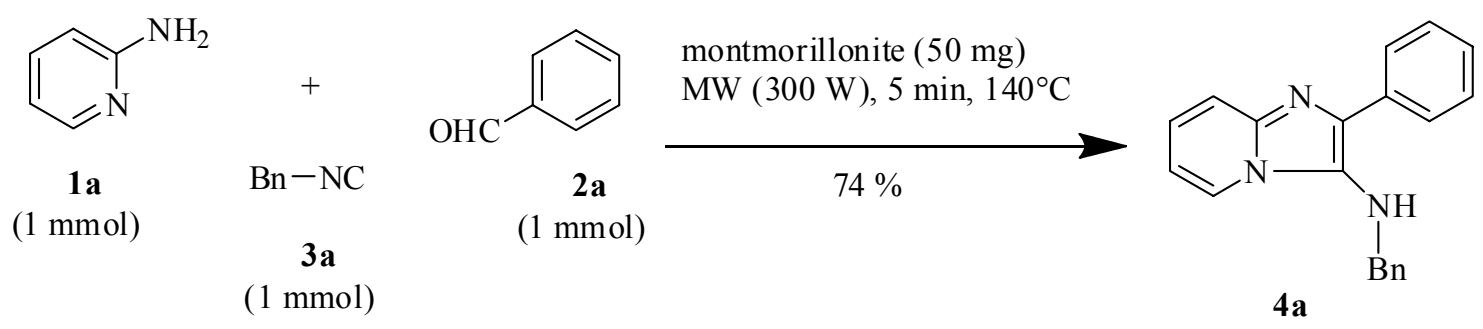

Scheme 1. Microwave-assisted synthesis of $\mathbf{4 a}$ under solvent-free conditions.

Further experiments demonstrated that the yield of $\mathbf{4 a}$ could be improved markedly when the reaction was run in an organic solvent (Table 1). Optimizing the reaction conditions with regard to the amounts of substrates, reaction time and temperature, solvent and the amount of montmorillonite revealed that 4 a could be isolated in $92 \%$ yield when $1 \mathrm{mmol} \mathrm{1a}, 1.09 \mathrm{mmol}$ of 2a and $1.25 \mathrm{mmol}$ of 3a were reacted with $76 \mathrm{mg}$ montmorillonite in $2 \mathrm{~mL}$ toluene at $160{ }^{\circ} \mathrm{C}$ for $7 \mathrm{~min}$ (Entry 7). When the reaction was run in a sealed vial under thermal conditions (oil bath) the yield of $4 \mathbf{a}$ dropped to $75 \%$.

The microwave-assisted synthesis of $\mathbf{4 a}$ was also studied in different ILs in the presence of montmorillonite. First, the reaction was performed with different imidazolium salts (Table 2). It was found that at higher reaction temperatures mainly decomposition occurs (Entries 3,5). But even at $90^{\circ} \mathrm{C}$ the formation of $\mathbf{4 a}$ was accompanied with the formation of a side product of unknown structure (Entries 1,2,6). The only IL allowing for the clean formation of $\mathbf{4 a}$ was [bmim] $\mathrm{BF}_{4}$ at $90^{\circ} \mathrm{C}$ (Entry 4). Under these conditions 4 a could be isolated with $70 \%$ yield. However, in no case did the yield of $\mathbf{4 a}$ exceed that obtained under the conditions given in Table 1 , Entry 7. 
Table 1. Optimization of the reaction conditions for the synthesis of $\mathbf{4 a}$ in organic solvents

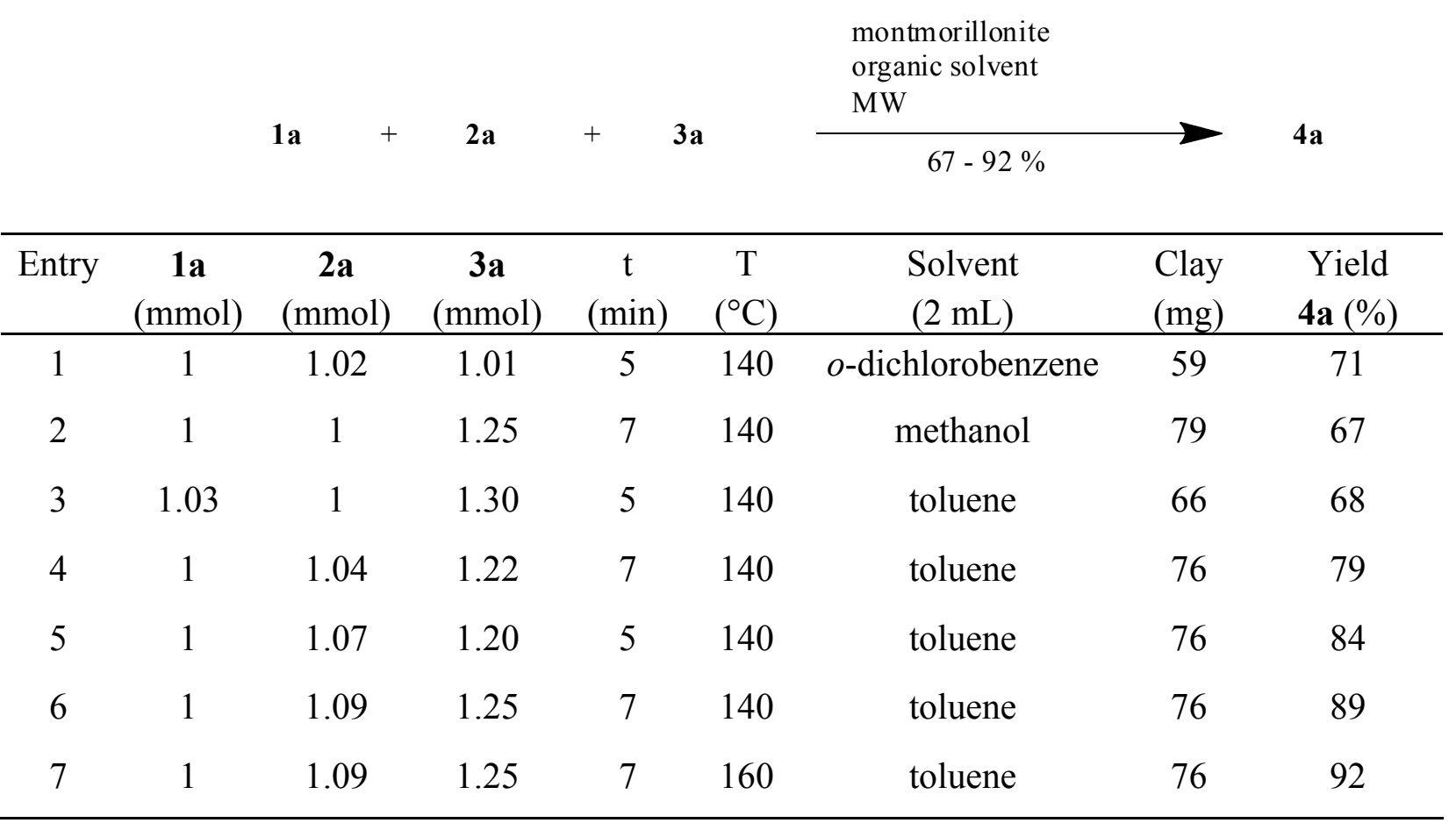

Table 2. The use of imidazolium salts as reaction medium in the synthesis of $\mathbf{4 a}$

$\underset{(1 \mathrm{mmol})}{\mathbf{1 a}}+\underset{(1.09 \mathrm{mmol})}{\mathbf{2 a}}+\underset{(1.25 \mathrm{mmol})}{\mathbf{3 a}} \frac{\mathrm{MW}}{\text { ionic liquid }(2 \mathrm{~mL})} \underset{\mathbf{4 a}}{\mathbf{m}}$

\begin{tabular}{cccccc}
\hline Entry & Ionic liquid & Power $(\mathrm{W})$ & $\mathrm{t}(\mathrm{min})$ & $\mathrm{T}\left({ }^{\circ} \mathrm{C}\right)$ & yield 4a $(\%)$ \\
\hline 1 & {$[\mathrm{bmim}] \mathrm{Cl}$} & 10 & 30 & 90 & $66^{1}$ \\
2 & {$[\mathrm{bmim}] \mathrm{Br}$} & 10 & 30 & 90 & $39^{1}$ \\
3 & {$[\mathrm{bmim}] \mathrm{Br}$} & 30 & 10 & 160 & Decomposition \\
4 & {$[\mathrm{bmim}] \mathrm{BF}_{4}$} & 10 & 30 & 90 & 70 \\
5 & {$\left[\mathrm{bmim}^{1} \mathrm{MeSO}_{4}\right.$} & 20 & 7 & 140 & Decomposition \\
6 & {$[\mathrm{bmim}] \mathrm{PF}_{6}$} & 20 & 7 & 140 & $39^{1}$ \\
\hline
\end{tabular}

${ }^{1}$ In addition, a side product of unknown structure was observed.

Furthermore, it was studied whether the imidazolium salts can be replaced by guanidinium salts. When the microwave-assisted reaction between 1a, 2a and 3a was run in guanidinium salt 5a as ionic liquid in the presence of montmorillonite $43 \%$ of $4 \mathbf{a}$ could be isolated (Table 3, Entry 1). In a final set of experiments the reaction was studied in the absence of montmorillonite. For this purpose 1 eq 1a, 1.09 eq $\mathbf{2 a}$ and 1.25 eq $\mathbf{3 a}$ were reacted under microwave conditions in 1 eq 
of different guanidinium salts 5a-d (Table 3, Structure Block 1). The product was formed in each of the ionic liquids employed, but the outcome strongly depends on the structure of the guanidinium salts employed. The highest yield (68\%) was obtained with 5c (Entry 4). However, in no case did the yield of $\mathbf{4 a}$ exceed that obtained under the conditions given in Table 1, Entry 7. The results clearly demonstrate that the three component reaction can be performed in guanidinium salts acting both as solvent and as catalyst. Further studies will be needed to study the influence of the structure of the guanidinium salts.

Table 3. The use of guanidinium salts for the synthesis of $\mathbf{4 a}$

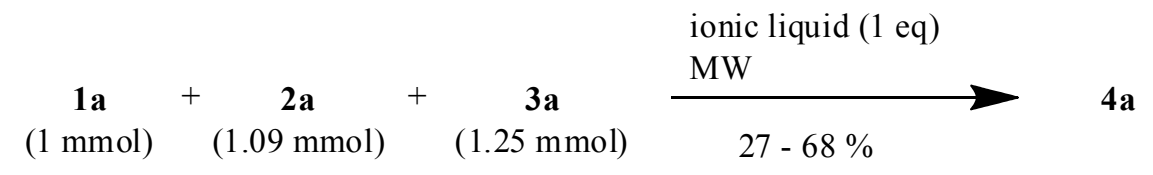

\begin{tabular}{ccccccc}
\hline Entry & Ionic liquid & montmorillonite & Power $(\mathrm{W})$ & $\mathrm{t}(\mathrm{min})$ & $\mathrm{T}\left({ }^{\circ} \mathrm{C}\right)$ & yield 4a $(\%)$ \\
\hline 1 & $\mathbf{5 a}^{1}$ & $76 \mathrm{mg}$ & 10 & 30 & 90 & 43 \\
2 & $\mathbf{5 a}$ & - & 20 & 7 & 160 & $48^{2}$ \\
3 & $\mathbf{5 a}$ & - & 10 & 90 & 90 & 29 \\
4 & $\mathbf{5 b}$ & - & 10 & 90 & 90 & 27 \\
5 & $\mathbf{5 c}$ & - & 10 & 90 & 90 & 68 \\
6 & $\mathbf{5 d}$ & - & 10 & 90 & 90 & 40 \\
\hline
\end{tabular}

${ }^{1}$ The reaction was performed in $2 \mathrm{~mL}$ of $\mathbf{5 a} .{ }^{2}$ In addition, a side product of unknown structure was observed.<smiles></smiles><smiles></smiles><smiles></smiles><smiles>C/C=C/CN(C)C(N(CC)CC)N(CC)CCC</smiles>

Structure Block 1. Structures of guanidinium salts 5a-d.

It turned out that the highest yield of $4 \mathbf{a}$ could be obtained when the microwave-assisted reaction between 1a, 2a and 3a using montmorillonite as a catalyst was run in toluene as the solvent. Therefore, the scope of the three-component reaction was studied under these reaction conditions. It was found that 2-aminopyridines $\mathbf{1} \mathbf{a}, \mathbf{b}$ can be successfully reacted with different aldehydes 2a-f and isocyanides 3a-c to produce a number of imidazo[1,2-a]pyridines 4a-j with yields ranging from $16 \%$ to $98 \%$ in analytically pure form (Table 4 , Structure Block 2 ). 
Table 4. Synthesis of imidazo[1,2-a]pyridines 4a-j from different 2-aminopyridines 1, aldehydes $\mathbf{2}$ and isocyanides $\mathbf{3}$

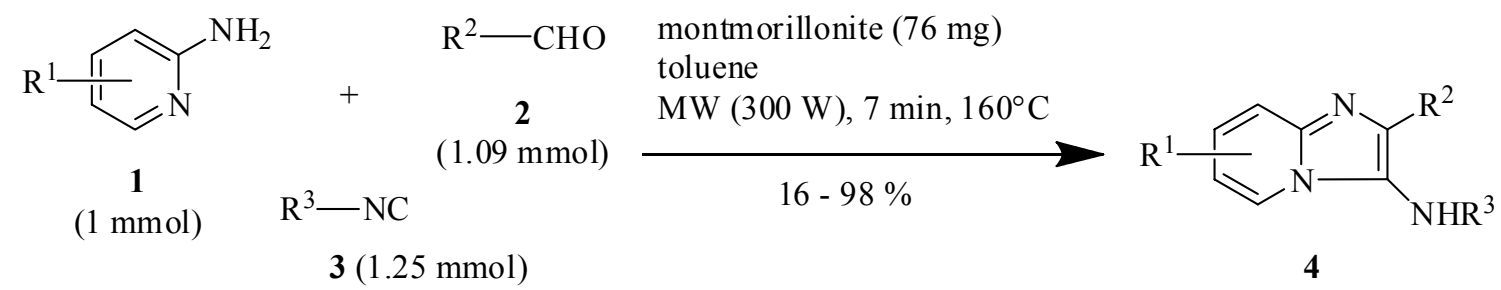

\begin{tabular}{|c|c|c|c|c|c|c|c|c|}
\hline Entry & 1 & $\mathrm{R}^{1}$ & 2 & $\mathrm{R}^{2}$ & 3 & $\mathrm{R}^{3}$ & Product & Yield $(\%)$ \\
\hline 1 & $1 \mathbf{a}$ & $\mathrm{H}$ & $\mathbf{2 a}$ & & $\mathbf{3 a}$ & 10 & $4 a$ & 92 \\
\hline 2 & $1 \mathbf{a}$ & $\mathrm{H}$ & $2 b$ & & $3 \mathbf{a}$ & 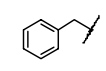 & $4 b$ & 70 \\
\hline 3 & $1 \mathbf{a}$ & $\mathrm{H}$ & $2 c$ & & 3a & ivy & $4 c$ & 80 \\
\hline 4 & $1 \mathbf{a}$ & $\mathrm{H}$ & $2 d$ & & $\mathbf{3 a}$ & & $4 d$ & 77 \\
\hline 5 & $1 \mathbf{a}$ & $\mathrm{H}$ & $2 e$ & & $\mathbf{3 a}$ & & $4 e$ & 60 \\
\hline 6 & $1 \mathbf{a}$ & $\mathrm{H}$ & $2 f$ & & $3 \mathbf{a}$ & 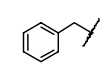 & 4f & 53 \\
\hline 7 & $1 \mathbf{a}$ & $\mathrm{H}$ & $\mathbf{2 a}$ & & $\mathbf{3 b}$ & & $4 g$ & 94 \\
\hline 8 & $1 b$ & $5-\mathrm{Br}$ & $\mathbf{2 a}$ & & $\mathbf{3 a}$ & y & $4 h$ & 72 \\
\hline 9 & $1 b$ & $5-\mathrm{Br}$ & $2 \mathrm{~g}$ & & $3 \mathbf{a}$ & y & $4 i$ & 16 \\
\hline 10 & $1 \mathbf{a}$ & $\mathrm{H}$ & $2 b$ & & $3 c$ & & $4 \mathbf{j}$ & 98 \\
\hline
\end{tabular}


<smiles>c1ccc(CNc2c(-c3ccccc3)nc3ccccn23)cc1</smiles>

$4 a$<smiles>Clc1ccc(-c2nc3ccccn3c2NCc2ccccc2)cc1</smiles>

4b<smiles>Brc1ccc(-c2nc3ccccn3c2NCc2ccccc2)cc1</smiles>

$4 c$<smiles>N#Cc1ccc(-c2nc3ccccn3c2NCc2ccccc2)cc1</smiles>

4d<smiles>COc1cc(-c2nc3ccccn3c2NCc2ccccc2)cc(OC)c1OC</smiles>

$4 e$<smiles>Brc1ccc2nc(-c3ccccc3)c(NCc3ccccc3)n2c1</smiles>

4h<smiles>O=[N+]([O-])c1ccccc1/C=C/c1nc2ccccn2c1NCc1ccccc1</smiles>

$4 f$<smiles>CCOC(=O)/C=C(\C)c1nc2ccc(Br)cn2c1NCc1ccccc1</smiles>

$4 i$<smiles>CC(C)CC(=O)Oc1ccccc1Nc1c(-c2ccccc2)nc2ccccn12</smiles><smiles>Clc1ccc(-c2nc3ccccn3c2NC2CCCCC2)cc1</smiles>

$4 j$

Structure Block 2. Structures of imidazo[1,2-a]pyridines $\mathbf{4 a - j}$.

The structures of all imidazo[1,2-a]pyridines $\mathbf{4 a - j}$ described here have been elucidated by means of mass, ${ }^{1} \mathrm{H},{ }^{13} \mathrm{C}, \mathrm{COSY}$, HSQC and HMBC spectroscopic methods. The assignment of the quaternary carbons relies on long-range correlations in the HMBC spectra.

\section{Conclusions}

In summary, the microwave-assisted three-component reaction between 2-aminopyridines, aldehydes and isocyanides with montmorillonite as the catalyst and in toluene as the solvent delivers 3-amino-substituted imidazo[1,2-a]pyridines with yields up to $98 \%$ within a few minutes. The organic solvent can be replaced by ILs like imidazolium or guanidinium salts. With guanidinium salts the reaction can be performed in the absence of any further catalyst. 


\section{Experimental Section}

General. Starting materials were purchased from chemical companies and used without purification. Toluene was distilled from sodium. All microwave-assisted reactions were performed using a Discover ${ }^{\mathrm{TM}}$ single mode cavity microwave synthesizer (CEM Corp.) producing continuous microwave irradiation at $2450 \mathrm{MHz}$. All experiments were conducted under argon. Thin-layer chromatography (TLC) was performed on TLC aluminum roll silica gel $60 \mathrm{~F}_{254}$ (Merck). Compounds were visualized with UV light $(\lambda=254 \mathrm{~nm})$ and/or immersion in $\mathrm{KMnO}_{4}$ solution followed by heating. Column chromatography was performed on silica gel MN $60,0.063 \mathrm{~mm}-0.200 \mathrm{~mm}$. Melting points were determined on a Kofler melting point apparatus (Reichert, Austria) and are uncorrected. IR (ATR) spectra were taken on a Spectrum One FT-IR spectrometer (Perkin Elmer). UV spectra were measured using a CARY 4E spectrophotometer (Varian). NMR spectra were recorded on a Varian Unity INOVA spectrometer $(300 / 75 \mathrm{MHz})$ in $\mathrm{CDCl}_{3}$; the ${ }^{1} \mathrm{H}$ and ${ }^{13} \mathrm{C}$ chemical shifts were referenced to residual solvent signals at $\delta_{\mathrm{H}}=7.26$ and $\delta_{\mathrm{C}}=77.0$ relative to TMS. Mass spectra were recorded on a MAT 90 with $70 \mathrm{eV}$ ionization energy (Finnigan MAT). Elemental analyses were carried out by F. Hambloch, Institute of Organic and Biomolecular Chemistry, University of Göttingen.

\section{General procedure $A$ for the reaction of 1, 2 and 3 under microwave conditions in toluene} 1 (1 mmol), $2(1.09 \mathrm{mmol})$ and $\mathbf{3}(1.25 \mathrm{mmol})$ were suspended in toluene $(2 \mathrm{~mL})$ and placed in a $10 \mathrm{~mL}$ reaction vial heated and cooled under Ar. After addition of montmorillonite $(76 \mathrm{mg})$ the vial was sealed with a septum and irradiated with microwaves $(300 \mathrm{~W})$ for $7 \mathrm{~min}$ at $160^{\circ} \mathrm{C}$. The reaction mixture was allowed to cool to r.t. and was then diluted with $\mathrm{CH}_{2} \mathrm{Cl}_{2}(10 \mathrm{~mL})$ and filtered. The residue obtained after concentration in vacuo was purified by column chromatography on silica gel to yield 4.

General procedure $B$ for the reaction of 1a, 2a and 3a under microwave conditions in imidazolium salts

1a $(1 \mathrm{mmol}), \mathbf{2 a}(1.09 \mathrm{mmol}), 3 \mathbf{3 a}(1.25 \mathrm{mmol})$ and montmorillonite $(76 \mathrm{mg})$ were placed in a 10 $\mathrm{mL}$ reaction vial that had been heated and cooled under Ar. After addition of the imidazolium salt $(2 \mathrm{~mL})$ the vial was sealed with a septum and irradiated with microwaves. After completion of the reaction, the mixture was allowed to cool to r.t. and washed with EE or TBME $(5 \times 5 \mathrm{~mL})$. The combined organic extracts were concentrated in vacuo and the residue was purified by column chromatography on silica gel to yield $\mathbf{4 a}$.

General procedure $C$ for the reaction of $1 \mathrm{a}, 2 \mathrm{a}$ and $3 \mathrm{a}$ under microwave conditions in guanidinium salts

1a $(1 \mathrm{mmol}), \mathbf{2 a}(1.09 \mathrm{mmol}), \mathbf{3 a}(1.25 \mathrm{mmol})$ and the guanidinium salt $\mathbf{5}(1 \mathrm{mmol})$ were placed in a $10 \mathrm{~mL}$ reaction vial that had been heated and cooled under argon. The vial was sealed with a 
septum and irradiated with microwaves. After completion of the reaction, the mixture was allowed to cool to r.t. and then purified by column chromatography on silica gel to yield $\mathbf{4 a}$.

N-Benzyl-2-phenylimidazo[1,2-a]pyridin-3-amine (4a). ${ }^{10 \mathrm{f}}$ According to general procedure A $94 \mathrm{mg}$ (1 mmol) 1a, $116 \mathrm{mg}(1.09 \mathrm{mmol}) \mathbf{2 a}$ and $146 \mathrm{mg}(1.25 \mathrm{mmol})$ 3a were reacted with 76 $\mathrm{mg}$ montmorillonite in $2 \mathrm{~mL}$ toluene. After work up and column chromatography $(\mathrm{TBME} / \mathrm{PE}=$ 10/2) $274 \mathrm{mg}(0.92 \mathrm{mmol}) \mathbf{4 a}(92 \%)$ were isolated as a yellow solid. mp $122-123^{\circ} \mathrm{C}$ (Lit. ${ }^{10 \mathrm{f}} \mathrm{mp} 112-113^{\circ} \mathrm{C}$ ). IR (ATR): $\widetilde{v}=3256,3057,3020,1564,1491,1472$, 1444, 1386, 1348, 1334, 1228, 1192, 1077, 1026, 907, 752, 735, 706, 694. ${ }^{1} \mathrm{H}$ NMR (300 MHz, $\left.\mathrm{CDCl}_{3}\right): \delta=3.53(1 \mathrm{H}, \mathrm{t}, J=6.2 \mathrm{~Hz}, \mathrm{NH}), 4.20\left(2 \mathrm{H}, \mathrm{d}, J=6.3 \mathrm{~Hz}, 7^{\text {“ ‘ }}-\mathrm{CH}_{2}\right), 6.74(1 \mathrm{H}, \mathrm{ddd}, J=$ $0.9 \mathrm{~Hz}, J=6.8 \mathrm{~Hz}, J=6.8 \mathrm{~Hz}, 5-\mathrm{H}), 7.13(1 \mathrm{H}, \mathrm{ddd}, J=1.3 \mathrm{~Hz}, J=6.7 \mathrm{~Hz}, J=9.2 \mathrm{~Hz}, 6-\mathrm{H})$, 7.27-7.38 (6H, m, 4'-H, 2“"-H, 3“'-H, 4“"-H, 5“'-H, 6“'-H), 7.45 (2H, t, J= 7.6 Hz, 3'-H, 5'-H), $7.56(1 \mathrm{H}, \mathrm{bd}, J=9.1 \mathrm{~Hz}, 7-\mathrm{H}), 7.90-8.05\left(3 \mathrm{H}, \mathrm{m}, 2^{6}-\mathrm{H}, 66-\mathrm{H}, 4-\mathrm{H}\right) .{ }^{13} \mathrm{C}-\mathrm{NMR}\left(75 \mathrm{MHz}, \mathrm{CDCl}_{3}\right)$ : $\delta=52.40\left(7^{،}{ }^{\prime}-\mathrm{CH}_{2}\right), 111.79$ (5-C), 117.31 (7-C), 122.35 (4-C), 124.20 (6-C), 125.62 (3-C),

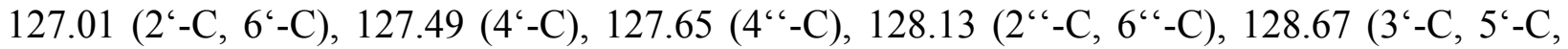

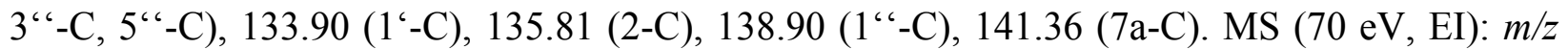
$(\%)=299(45)\left[\mathrm{M}^{+}\right], 208$ (100), 181 (97), 91 (13), 78 (87), 51 (11).

2-(4-Chlorophenyl)- $N$-benzylimidazo[1,2-a]pyridin-3-amine (4b). According to the general procedure A $94 \mathrm{mg}$ (1 mmol) 1a, $153 \mathrm{mg}(1.09 \mathrm{mmol}) \mathbf{2 b}$ and $146 \mathrm{mg}(1.25 \mathrm{mmol}) \mathbf{3 a}$ were reacted with $76 \mathrm{mg}$ montmorillonite in $2 \mathrm{~mL}$ toluene. After work up and column chromatography $(\mathrm{TBME} / \mathrm{PE}=5 / 3) 233 \mathrm{mg}(0.7 \mathrm{mmol}) \mathbf{4 b}(70 \%)$ were isolated as a yellow solid.

mp 166-167 ${ }^{\circ} \mathrm{C}$. IR (ATR): $\widetilde{v}=3249,3018,2919,1558,1488,1454,1384,1350,1332,1242$, $1188,1090,1076,1010,911,835,750,732,698$. UV $\left(\mathrm{CH}_{3} \mathrm{CN}\right): \lambda_{\max }(\lg \varepsilon)=339 \mathrm{~nm}(3.89), 253$ (4.58). ${ }^{1} \mathrm{H}-\mathrm{NMR}\left(300 \mathrm{MHz}, \mathrm{CDCl}_{3}\right): \delta=3.42(1 \mathrm{H}, \mathrm{t}, J=5.9 \mathrm{~Hz}, \mathrm{NH}), 4.17(2 \mathrm{H}, \mathrm{d}, J=5.9 \mathrm{~Hz}$, 7 “ $\left.-\mathrm{CH}_{2}\right), 6.74(1 \mathrm{H}, \mathrm{ddd}, J=1.1 \mathrm{~Hz}, J=6.7 \mathrm{~Hz}, J=6.7 \mathrm{~Hz}, 5-\mathrm{H}), 7.13(1 \mathrm{H}, \mathrm{ddd}, J=1.3 \mathrm{~Hz}, J=$ $6.6 \mathrm{~Hz}, J=9.1 \mathrm{~Hz}, 6-\mathrm{H}), 7.28-7.35\left(5 \mathrm{H}, \mathrm{m}, 2^{\prime c}-\mathrm{H}, 3^{\prime c}-\mathrm{H}, 4^{\prime c}-\mathrm{H}, 5^{\prime c}-\mathrm{H}, 6\right.$ 6“- $\left.\mathrm{H}\right), 7.37-7.41(2 \mathrm{H}, \mathrm{m}$, $\left.3^{`}-\mathrm{H}, 5^{6}-\mathrm{H}\right), 7.53(1 \mathrm{H}, \mathrm{dt}, J=1.1 \mathrm{~Hz}, J=9.1 \mathrm{~Hz}, 7-\mathrm{H}), 7.96\left(2 \mathrm{H}, \mathrm{m}, 2^{`}-\mathrm{H}, 6^{6}-\mathrm{H}\right), 7.98$ (1H, dt, $J$ $=1.4 \mathrm{~Hz}, J=6.7 \mathrm{~Hz}, 4-\mathrm{H}) .{ }^{13} \mathrm{C}-\mathrm{NMR}\left(75 \mathrm{MHz}, \mathrm{CDCl}_{3}\right): \delta=52.39\left(7^{\text {‘ }}-\mathrm{CH}_{2}\right), 111.85$ (5-C), 117.48 (7-C), 122.27 (4-C), 124.28 (6-C), 125.52 (3-C), 127.74 (4“'-C), 128.15 (2'-C, 6'-C), 128.20 and 128.74 (2' '-C and 6' -C or 3' '-C and 5' '-C), 128.76 (3'-C, 5'-C), 132.68 (1'-C), 133.17 (4'-C), 135.15 (2-C), 138.78 (1'’-C), 141.60 (7a-C). MS (70 eV, EI): m/z (\%) = 333 (16) $\left[\mathrm{M}^{+}\right], 242$ (100), 215 (71), 78 (40). Anal. Calcd. for $\mathrm{C}_{20} \mathrm{H}_{16} \mathrm{ClN}_{3}$ : C, 71.96; H, 4.83; Cl, 10.62; N, 12.59. Found: C, 71.82; H, 4.74; N, 12.80 .

$\mathrm{N}$-Benzyl-2-(4-bromophenyl)imidazo[1,2-a]pyridin-3-amine (4c). According to the general procedure A $94 \mathrm{mg}$ (1 mmol) 1a, $202 \mathrm{mg}$ (1.09 mmol) 2c and $146 \mathrm{mg}(1.25 \mathrm{mmol})$ 3a were reacted with $76 \mathrm{mg}$ montmorillonite in $2 \mathrm{~mL}$ toluene. After work up and column chromatography $(\mathrm{TBME} / \mathrm{PE}=5 / 2) 304 \mathrm{mg}(0.8 \mathrm{mmol}) \mathbf{4 c}(80 \%)$ were isolated as a pale yellow solid. 


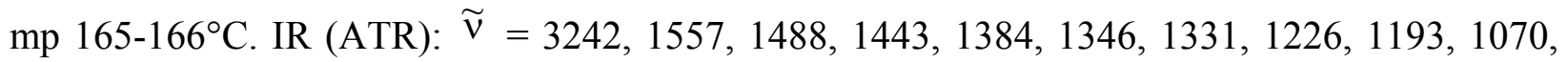
$1008,829,752,733,695$. UV $\left(\mathrm{CH}_{3} \mathrm{CN}\right): \lambda_{\max }(\lg \varepsilon)=340 \mathrm{~nm}$ (3.93), 255 (4.59). ${ }^{1} \mathrm{H}-\mathrm{NMR}(300$ $\left.\mathrm{MHz}, \mathrm{CDCl}_{3}\right): \delta=3.42(1 \mathrm{H}, \mathrm{t}, J=6.0 \mathrm{~Hz}, \mathrm{NH}), 4.17\left(2 \mathrm{H}, \mathrm{d}, J=6.2 \mathrm{~Hz}, 7\right.$ “ $\left.-\mathrm{CH}_{2}\right), 6.75(1 \mathrm{H}$, ddd, $J=1.1 \mathrm{~Hz}, J=6.8 \mathrm{~Hz}, J=6.8 \mathrm{~Hz}, 5-\mathrm{H}), 7.14(1 \mathrm{H}, \mathrm{ddd}, J=1.3 \mathrm{~Hz}, J=6.7 \mathrm{~Hz}, J=9.0 \mathrm{~Hz}, 6-\mathrm{H})$, 7.27-7.35 (5H, m, 2''-H, 3''-H, 4'-H, 5' '-H, 6'--H), 7.54 (3H, overlapped, 3'-H, 5'-H, 7-H), $7.87\left(2 \mathrm{H}, \mathrm{d}, J=8.5 \mathrm{~Hz}, 2^{\prime}-\mathrm{H}, 6{ }^{\prime}-\mathrm{H}\right), 7.95(1 \mathrm{H}, \mathrm{dt}, J=1.2 \mathrm{~Hz}, J=6.9 \mathrm{~Hz}, 4-\mathrm{H}) .{ }^{13} \mathrm{C}-\mathrm{NMR}(75$ $\left.\mathrm{MHz}, \mathrm{CDCl}_{3}\right): \delta=52.32$ (7“'- $\left.\mathrm{CH}_{2}\right), 112.05$ (5-C), 117.28 (7-C), 121.51 (4'-C), 122.35 (4-C), 124.64 (6-C), 125.61 (3-C), 127.76 (4''-C), 128.15 and 128.48 and 128.74 (2'-C and 6'-C or 2''$\mathrm{C}$ and 6"-C or 3"'-C and 5'-C), 131.71 (3'-C, 5'-C), 132.76 (1'-C), 134.79 (2-C), 138.73 (1'"C), 141.39 (7a-C). MS (70 eV, EI): $m / z(\%)=377(26)\left[\mathrm{M}^{+}\right], 286$ (100), 259 (64), 180 (9), 91 (19), 78 (80). Anal. Calcd. for $\mathrm{C}_{20} \mathrm{H}_{16} \mathrm{BrN}_{3}: \mathrm{C}, 63.50 ; \mathrm{H}, 4.26$; $\mathrm{Br}, 21.12 ; \mathrm{N}, 11.11$. Found: $\mathrm{C}$, 63.55; H, 4.19; N, 11.02 .

$N$-Benzyl-2-(4-cyanphenyl)imidazo[1,2-a]pyridin-3-amine (4d). According to the general procedure A $94 \mathrm{mg}(1 \mathrm{mmol}) \mathbf{1 a}, 143 \mathrm{mg}(1.09 \mathrm{mmol}) \mathbf{2 d}$ and $146 \mathrm{mg}(1.25 \mathrm{mmol})$ 3a were reacted with $76 \mathrm{mg}$ montmorillonite in $2 \mathrm{~mL}$ toluene. After work up and column chromatography $(\mathrm{TBME} / \mathrm{PE}=10 / 3) 249 \mathrm{mg}(0.77 \mathrm{mmol}) \mathbf{4 d}(77 \%)$ were isolated as a yellow solid.

mp 190-191 ${ }^{\circ} \mathrm{C}$. IR (ATR): $\widetilde{v}=3244,2225,1605,1574,1454,1348,1190,851,760,748,729$, 701. UV $\left(\mathrm{CH}_{3} \mathrm{CN}\right): \lambda_{\max }(\lg \varepsilon)=350 \mathrm{~nm}(4.00), 265$ (4.53). ${ }^{1} \mathrm{H}-\mathrm{NMR}\left(300 \mathrm{MHz}, \mathrm{CDCl}_{3}\right): \delta=$ $3.54(1 \mathrm{H}, \mathrm{t}, J=6.0 \mathrm{~Hz}, \mathrm{NH}), 4.18\left(2 \mathrm{H}, \mathrm{d}, J=6.1 \mathrm{~Hz}, 7\right.$ ' $\left.-\mathrm{CH}_{2}\right), 6.77(1 \mathrm{H}, \mathrm{ddd}, J=1.1 \mathrm{~Hz}, J=$ $6.8 \mathrm{~Hz}, J=6.8 \mathrm{~Hz}, 5-\mathrm{H}), 7.18(1 \mathrm{H}, \mathrm{ddd}, J=1.3 \mathrm{~Hz}, J=6.6 \mathrm{~Hz}, J=9.0 \mathrm{~Hz}, 6-\mathrm{H}), 7.27-7.31(5 \mathrm{H}$, m, 2''-H, 3''-H, 4"'-H, 5' '-H, 6'-H), 7.54 (1H, dt, $J=1,1 \mathrm{~Hz}, J=8.9 \mathrm{~Hz}, 7-\mathrm{H}), 7.65$ (2H, d, $J=$ $8.4 \mathrm{~Hz}, 3$ '-H, 5'-H), 7.93 (1H, dt, $J=1.2 \mathrm{~Hz}, J=6.8 \mathrm{~Hz}, 4-\mathrm{H}), 8.12\left(2 \mathrm{H}, \mathrm{d}, J=8.4 \mathrm{~Hz}, 2^{\prime}-\mathrm{H}, 6^{\prime}-\right.$ H). ${ }^{13} \mathrm{C}-\mathrm{NMR}\left(75 \mathrm{MHz}, \mathrm{CDCl}_{3}\right): \delta=52.40\left(7^{\prime}{ }^{-}-\mathrm{CH}_{2}\right), 110.43\left(4^{\prime}-\mathrm{C}\right), 112.35(5-\mathrm{C}), 117.62(7-\mathrm{C})$, 119.08 (1'-C), 122.36 (4-C), 125.09 (6-C), 126.70 (3-C), 127.11 (2'-C, 6'-C), 127.89 (4'”-C), 128.13 (2"'-C, 6"-C), 128.80 (3'”-C, 5''-C), 132.29 (3'-C, 5'-C), 133.90 (2-C), 138.47 (1'”-C),

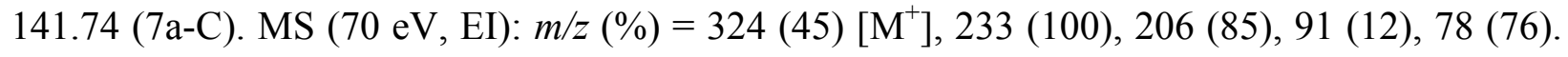
HRMS (70 eV, EI) for $\mathrm{C}_{21} \mathrm{H}_{16} \mathrm{~N}_{4}$ : Calcd.: 324.1375; Found: 324.1397 .

$\mathrm{N}$-Benzyl-2-(3,4,5-trimethoxyphenyl)imidazo[1,2-a]pyridin-3-amine (4e). According to the general procedure A $94 \mathrm{mg}(1 \mathrm{mmol}) \mathbf{1 a}, 214 \mathrm{mg}(1.09 \mathrm{mmol}) \mathbf{2 e}$ and $146 \mathrm{mg}(1.25 \mathrm{mmol}) \mathbf{3 a}$ were reacted with $76 \mathrm{mg}$ montmorillonite in $2 \mathrm{~mL}$ toluene. After work up and column chromatography $(\mathrm{EE} / \mathrm{PE}=5 / 2) 232 \mathrm{mg}(0.6 \mathrm{mmol}) \mathbf{4 e}(60 \%)$ were isolated as a pale yellow solid.

mp 131-132 ${ }^{\circ} \mathrm{C}$. IR (ATR): $\widetilde{v}=3189,2829,1587,1495,1446,1412,1391,1357,1231,1120$, 1000, 759, 723, 694. UV $\left(\mathrm{CH}_{3} \mathrm{CN}\right): \lambda_{\max }(\lg \varepsilon)=339 \mathrm{~nm}$ (3.89), 261 (4.43), 223 (4.59). ${ }^{1} \mathrm{H}-\mathrm{NMR}$ $\left(300 \mathrm{MHz}, \mathrm{CDCl}_{3}\right): \delta=3.46(1 \mathrm{H}, \mathrm{bs}, \mathrm{NH}), 3.90\left(9 \mathrm{H}, \mathrm{s}, 3^{\circ}-\mathrm{OCH}_{3}, 4^{6}-\mathrm{OCH}_{3}, 5^{\circ}-\mathrm{OCH}_{3}\right), 4.23(2 \mathrm{H}$, d, $J=5.6 \mathrm{~Hz}, 7$ “ $\left.-\mathrm{CH}_{2}\right), 6.75(1 \mathrm{H}$, ddd, $J=1.1 \mathrm{~Hz}, J=6.7 \mathrm{~Hz}, J=6.7 \mathrm{~Hz}, 5-\mathrm{H}), 7.13(1 \mathrm{H}$, ddd, $J$

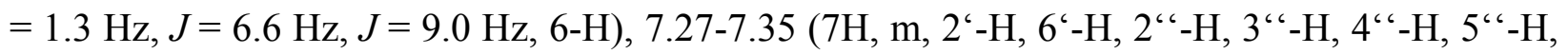
6 6“-H), $7.55(1 \mathrm{H}, \mathrm{dt}, J=1.1 \mathrm{~Hz}, J=9.1 \mathrm{~Hz}, 7-\mathrm{H}), 7.95(1 \mathrm{H}, \mathrm{dt}, J=1.2 \mathrm{~Hz}, J=6.8 \mathrm{~Hz}, 4-\mathrm{H}) .{ }^{13} \mathrm{C}-$ 
NMR (75 MHz, $\left.\mathrm{CDCl}_{3}\right): \delta=52.40\left(7^{\text {‘ }}-\mathrm{CH}_{2}\right), 56.22\left(3^{`}-\mathrm{OCH}_{3}, 5^{6}-\mathrm{OCH}_{3}\right), 60.91\left(4^{6}-\mathrm{OCH}_{3}\right)$, 104.26 (2‘-C, 6'-C), 111.89 (5-C), 117.23 (7-C), 122.20 (4-C), 124.26 (6-C), 125.28 (3-C), 127.73 (4“"-C), 128.02 (2“"-C, 6“"-C), 128.73 (3“"-C, 5“'-C), 129.57 (1'-C), 135.67 (2-C), 137.67 (4`-C), 138.88 (1“‘-C), 141.22 (7a-C), 153.40 (3'-C, 5‘-C). MS (70 eV, EI): m/z (\%) = 389 (25) $\left[\mathrm{M}^{+}\right.$], 298 (100), 271 (72), 78 (16). HRMS (70 eV, EI) for $\mathrm{C}_{23} \mathrm{H}_{23} \mathrm{~N}_{3} \mathrm{O}_{3}$ : Calcd.: 389.1700; Found: 389.1747.

N-Benzyl-2-(2-nitrophenylethenyl)imidazo[1,2-a]pyridin-3-amine (4f). According to the general procedure A $94 \mathrm{mg}$ (1 mmol) 1a, $193 \mathrm{mg}(1.09 \mathrm{mmol}) \mathbf{2 f}$ and $146 \mathrm{mg}$ (1.25 mmol) 3a were reacted with $76 \mathrm{mg}$ montmorillonite in $2 \mathrm{~mL}$ toluene. After work up and column chromatography $(\mathrm{EE} / \mathrm{PE}=5 / 3) 195 \mathrm{mg}(0.53 \mathrm{mmol}) \mathbf{4 f}(53 \%)$ were isolated as an orange solid.

mp 152-154 ${ }^{\circ} \mathrm{C}$. IR (ATR): $\widetilde{v}=3351,2929,1514,1348,1276,958,755,740,703$. UV $\left(\mathrm{CH}_{3} \mathrm{CN}\right): \lambda_{\max }(\lg \varepsilon)=375 \mathrm{~nm}(4.07), 317$ (4.14), 261 (4.44). ${ }^{1} \mathrm{H}-\mathrm{NMR}\left(300 \mathrm{MHz}, \mathrm{CDCl}_{3}\right): \delta=$ $3.48(1 \mathrm{H}, \mathrm{t}, J=6.8 \mathrm{~Hz}, \mathrm{NH}), 4.22\left(2 \mathrm{H}, \mathrm{d}, J=6.0 \mathrm{~Hz}, 7^{\prime \prime}-\mathrm{CH}_{2}\right), 6.74(1 \mathrm{H}, \mathrm{td}, J=1.1 \mathrm{~Hz}, J=6.8$ $\mathrm{Hz}, 5-\mathrm{H}), 6.87\left(1 \mathrm{H}, \mathrm{d}, J=15.8 \mathrm{~Hz}, 1^{\circ}-\mathrm{H}\right), 7.14(1 \mathrm{H}, \mathrm{ddd}, J=1.3 \mathrm{~Hz}, J=6.7 \mathrm{~Hz}, J=9.2 \mathrm{~Hz}, 6-$ H), 7.28-7.32 (5H, m, 2“"-H, 3“"-H, 4“"-H, 5“"-H, 6“"-H), 7.33-7.39 (1H, m, 6“-H), 7.51 (1H, dt, $J$ $=1.1 \mathrm{~Hz}, J=9.1 \mathrm{~Hz}, 7-\mathrm{H}), 7.53\left(2 \mathrm{H}\right.$, overlapped, $\left.7^{`}-\mathrm{H}, 8^{`}-\mathrm{H}\right), 7.85\left(1 \mathrm{H}, \mathrm{d}, J=15.8 \mathrm{~Hz}, 2^{`}-\mathrm{H}\right)$, $7.90\left(1 \mathrm{H}, \mathrm{dt}, J=0.9 \mathrm{~Hz}, J=8.3 \mathrm{~Hz}, 5^{`}-\mathrm{H}\right), 7.93(1 \mathrm{H}, \mathrm{dt}, J=1.1 \mathrm{~Hz}, J=7.0 \mathrm{~Hz}, 4-\mathrm{H}) .{ }^{13} \mathrm{C}-\mathrm{NMR}$ (75 MHz, $\left.\mathrm{CDCl}_{3}\right): \delta=53.08\left(7^{\prime c}-\mathrm{CH}_{2}\right), 111.75$ (5-C), 117.46 (7-C), 122.21 (4-C), 123.93 (2‘-C),

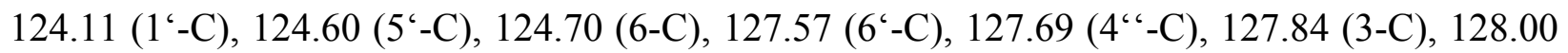

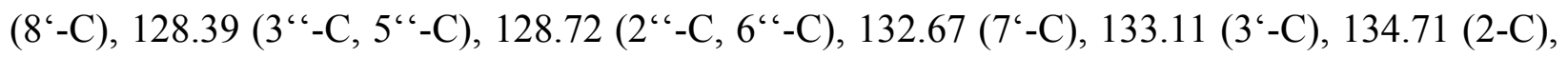

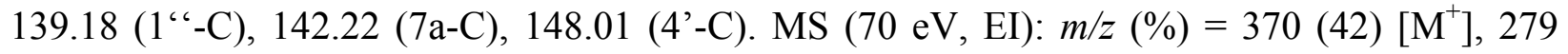
(100), 252 (46), 234 (16), 205 (10), 145 (7), 128 (6), 91 (7), 78 (41). HRMS (70 eV, EI) for $\mathrm{C}_{22} \mathrm{H}_{18} \mathrm{~N}_{4} \mathrm{O}_{2}$ : Calcd.: 370.1430 ; Found: 370.1426 .

\section{N-(2-(2,2-Dimethyl-1-oxopropoxy)phenyl)-2-phenylimidazo[1,2-a]pyridin-3-amine (4g).}

According to the general procedure A $94 \mathrm{mg}(1 \mathrm{mmol}) \mathbf{1 a}, 116 \mathrm{mg}(1.09 \mathrm{mmol}) \mathbf{2 a}$ and $254 \mathrm{mg}$ (1.25 mmol) $3 \mathbf{b}$ were reacted with $76 \mathrm{mg}$ montmorillonite in $2 \mathrm{~mL}$ toluene. After work up and column chromatography $(\mathrm{TBME} / \mathrm{PE}=4 / 3) 362 \mathrm{mg}(0.94 \mathrm{mmol}) \mathbf{4 g}(94 \%)$ were isolated as a pale gray solid.

mp 216-217 ${ }^{\circ} \mathrm{C}$. IR (ATR): $\widetilde{v}=2970,1743,1519,1492,1447,1391,1343,1254,1172,1106$, 756, 741, 699. UV $\left(\mathrm{CH}_{3} \mathrm{CN}\right): \lambda_{\max }(\lg \varepsilon)=330 \mathrm{~nm}$ (3.83), 245 (4.60), 202 (4.60). ${ }^{1} \mathrm{H}-\mathrm{NMR}(300$ $\left.\mathrm{MHz}, \mathrm{CDCl}_{3}\right): \delta=1.43\left(9 \mathrm{H}, \mathrm{s}, 2^{\text {“ }}\right.$ - $\left.-\left(\mathrm{CH}_{3}\right)_{3}\right), 5.63(1 \mathrm{H}, \mathrm{s}, \mathrm{NH}), 6.32(1 \mathrm{H}, \mathrm{dd}, J=1.3 \mathrm{~Hz}, J=7.9$ $\mathrm{Hz}, 5$ ' '-H), $6.80(1 \mathrm{H}, \mathrm{t}, J=6.8 \mathrm{~Hz}, 5-\mathrm{H}), 6.88(1 \mathrm{H}, \mathrm{td}, J=1.4 \mathrm{~Hz}, J=7.6 \mathrm{~Hz}, 3$ “'-H), 6.98 (1H, $\mathrm{td}, J=1.2 \mathrm{~Hz}, J=7.8 \mathrm{~Hz}, 4$ “'-H), $7.11\left(1 \mathrm{H}, \mathrm{dd}, J=1.3 \mathrm{~Hz}, J=7.9 \mathrm{~Hz}, 2^{\text {‘ }}-\mathrm{H}\right), 7.20-7.32$ (2H, m, $\left.4^{6}-\mathrm{H}, 6-\mathrm{H}\right), 7.38$ (2H, t, $\left.J=7.4 \mathrm{~Hz}, 3^{6}-\mathrm{H}, 5^{6}-\mathrm{H}\right), 7.66(1 \mathrm{H}, \mathrm{d}, J=9.1 \mathrm{~Hz}, 7-\mathrm{H}), 7.87$ (1H, d, $J=$ $6.9 \mathrm{~Hz}, 4-\mathrm{H}), 8.02\left(2 \mathrm{H}, \mathrm{d}, J=7.4 \mathrm{~Hz}, 2^{6}-\mathrm{H}, 66^{6}-\mathrm{H}\right) .{ }^{13} \mathrm{C}-\mathrm{NMR}\left(75 \mathrm{MHz}, \mathrm{CDCl}_{3}\right): \delta=27.31$ (2“، $\left.\left(\mathrm{CH}_{3}\right)_{3}\right), 39.44$ (2،“'-C), 112.45 (5-C), 113.79 (5“'-C), 116.97 (3-C), 117.65 (7-C), 120.14 (3“"C), 122.67 (2“'-C), 122.84 (4-C), 125.18 (6-C), 126.88 (2‘-C, 6'-C), 127.09 (4“'-C), 127.95 (4‘C), 128.67 (3‘-C, $\left.5^{6}-\mathrm{C}\right), 133.15$ (1'-C), 136.53 (6“'-C), 138.66 (1“'-C), 139.46 (2-C), 142.84 (7a- 
C), 176.77 (1','-C). MS (70 eV, EI): $m / z(\%)=385(100)\left[\mathrm{M}^{+}\right], 300$ (49), 181 (77), 78 (31), 57 (20). Anal. Calcd. for $\mathrm{C}_{24} \mathrm{H}_{23} \mathrm{~N}_{3} \mathrm{O}_{2}$ : C, 74.78; H, 6.01; N, 10.90; O, 8.30. Found: C, 74.81; H, $5.87 ; \mathrm{N}, 10.73$.

N-Benzyl-5-bromo-2-phenylimidazo[1,2-a]pyridin-3-amine (4h). According to the general procedure A $173 \mathrm{mg}$ (1 mmol) 1b, $116 \mathrm{mg}(1.09 \mathrm{mmol}) \mathbf{2 a}$ and $146 \mathrm{mg}(1.25 \mathrm{mmol})$ 3a were reacted with $76 \mathrm{mg}$ montmorillonite in $2 \mathrm{~mL}$ toluene. After work up and column chromatography $\left(\mathrm{CH}_{2} \mathrm{Cl}_{2} / \mathrm{EE}=12 / 1\right) 270 \mathrm{mg}(0.72 \mathrm{mmol}) \mathbf{4 h}(72 \%)$ were isolated as a white solid.

mp $173-174^{\circ} \mathrm{C}$. IR (ATR): $\widetilde{v}=3241,1474,1445,1404,1323,1215,1054,914,805,767,752$, 702, 693. UV ( $\left.\mathrm{CH}_{3} \mathrm{CN}\right): \lambda_{\max }(\lg \varepsilon)=347 \mathrm{~nm}$ (3.82), 251 (4.56). ${ }^{1} \mathrm{H}-\mathrm{NMR}\left(300 \mathrm{MHz}, \mathrm{CDCl}_{3}\right): \delta$ $=3.54(1 \mathrm{H}, \mathrm{t}, J=5.7 \mathrm{~Hz}, \mathrm{NH}), 4.18\left(2 \mathrm{H}, \mathrm{d}, J=5.9 \mathrm{~Hz}, 7^{\text {‘ }}-\mathrm{CH}_{2}\right), 7.16(1 \mathrm{H}, \mathrm{dd}, J=1.8 \mathrm{~Hz}, J=$

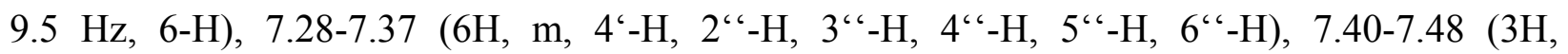
overlapped, 3'-H, 5'-H, 7-H), 7.95 (2H, d, $\left.J=7.3 \mathrm{~Hz}, 2^{6}-\mathrm{H}, 6^{6}-\mathrm{H}\right), 8.03$ (1H, dd, $J=0.8 \mathrm{~Hz}, J=$ $1.9 \mathrm{~Hz}, 4-\mathrm{H}) .{ }^{13} \mathrm{C}-\mathrm{NMR}\left(75 \mathrm{MHz}, \mathrm{CDCl}_{3}\right): \delta=52.49\left(7^{\text {‘ }}-\mathrm{CH}_{2}\right), 106.67$ (5-C), 117.96 (7-C), 122.66 (4-C), 125.84 (3-C), 126.99 (2‘-C, 6'-C), 127.50 (6-C), 127.80 (4`-C), 127.86 (4“" $-\mathrm{C})$, 128.23 (2“"-C, 6“"-C), 128.74 (3'-C, 5'-C, 3“'-C, 5“'-C), 133.50 (1'-C), 136.99 (2-C), 138.60 (1“'-C), 159.75 (7a-C). MS (70 eV, EI): $m / z(\%)=377$ (26) $\left[\mathrm{M}^{+}\right], 286$ (99), 259 (100), 180 (9), 156 (33), 103 (9), 91 (22), 76 (12). Anal. Calcd. for $\mathrm{C}_{20} \mathrm{H}_{16} \mathrm{BrN}_{3}$ : C, 63.50; H, 4.26; $\mathrm{Br}, 21.12$; N, 11.11. Found: C, 63.49; H, 4.06; N, 10.95 .

\section{$N$-Benzyl-5-bromo-2-(3-ethoxy-1-methyl-3-oxo-1-propen-1-yl)imidazo[1,2-a]pyridin-3- amine (4i).}

According to the general procedure A $173 \mathrm{mg}(1 \mathrm{mmol}) \mathbf{1 b}, 155 \mathrm{mg}(1.09 \mathrm{mmol}) \mathbf{2 g}$ and $146 \mathrm{mg}$ (1.25 mmol) 3a were reacted with $76 \mathrm{mg}$ montmorillonite in $2 \mathrm{~mL}$ toluene. After work up and column chromatography $\left(\mathrm{CH}_{2} \mathrm{Cl}_{2} / \mathrm{TBME}=15 / 1\right) 67 \mathrm{mg}(0.16 \mathrm{mmol}) 4 \mathbf{i}(16 \%)$ were isolated as a yellow solid.

mp $185-186^{\circ} \mathrm{C}$. IR (ATR): $\widetilde{v}=3303,1687,1614,1451,1331,1311,1197,1169,1105,1048$, 881, 812, 698. UV $\left(\mathrm{CH}_{3} \mathrm{CN}\right): \lambda_{\max }(\lg \varepsilon)=357 \mathrm{~nm}$ (3.84), 261 (4.49). ${ }^{1} \mathrm{H}-\mathrm{NMR}(300 \mathrm{MHz}$, $\left.\mathrm{CDCl}_{3}\right): \delta=1.32\left(3 \mathrm{H}, \mathrm{t}, J=7.1 \mathrm{~Hz}, 1^{\prime \prime}-\mathrm{CH}_{3}\right), 2.65\left(3 \mathrm{H}, \mathrm{s}, 1^{\circ}-\mathrm{CH}_{3}\right), 3.53(1 \mathrm{H}, \mathrm{t}, J=6.1 \mathrm{~Hz}, \mathrm{NH})$, $4.15(2 \mathrm{H}, \mathrm{d}, J=6.1 \mathrm{~Hz}, 7$ ', '-CH$), 4.22\left(2 \mathrm{H}, \mathrm{q}, J=7.1 \mathrm{~Hz}, 1\right.$ ' $\left.-\mathrm{CH}_{2}\right), 6.53\left(1 \mathrm{H}, \mathrm{s}, 2^{\prime}-\mathrm{H}\right), 7.17$ (1H, dd, $J=1.7 \mathrm{~Hz}, J=9.5 \mathrm{~Hz}, 6-\mathrm{H}), 7.29-7.35$ (5H, m, 2' ' -H, 3' '-H, 4' '-H, 5' '-H, 6', '-H), $7.39(1 \mathrm{H}, \mathrm{d}, J=9.5 \mathrm{~Hz}, 7-\mathrm{H}), 8.02(1 \mathrm{H}, \mathrm{s}, 4-\mathrm{H}) .{ }^{13} \mathrm{C}-\mathrm{NMR}\left(75 \mathrm{MHz}, \mathrm{CDCl}_{3}\right): \delta=14.34\left(1^{\prime \prime}-\right.$ $\left.\mathrm{CH}_{3}\right), 16.86\left(1^{\prime}-\mathrm{CH}_{3}\right), 52.55\left(7^{\prime}{ }^{\prime \prime}{ }^{-}-\mathrm{CH}_{2}\right), 59.81\left(1^{\prime}{ }^{\prime}-\mathrm{CH}_{2}\right), 107.16$ (5-C), 117.47 (2'-C), 118.36 (7-C), 122.68 (4-C), 127.54 (3-C), 128.01 (6-C or 4', '-C), 128.12 (6-C or 4','-C), 128.32 (2', 'C, 6','-C), 128.81 (3'”'-C, 5','-C), 137.53 (2-C), 138.12 (1','-C), 139.35 (7a-C), 148.45 (1'-C),

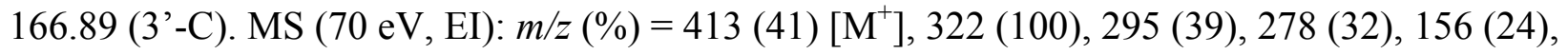
91 (38). Anal. Calcd. for $\mathrm{C}_{20} \mathrm{H}_{20} \mathrm{BrN}_{3} \mathrm{O}_{2}$ : C, 57.98; H, 4.87; Br, 19.29; N, 10.14; O, 7.72. Found: C, 57.85; H, 4.58; N, 9.88. 
2-(4-Chlorophenyl)- $N$-cyclohexylimidazo[1,2-a]pyridin-3-amine $(\mathbf{4 j}){ }^{10 a}$ According to the general procedure A $94 \mathrm{mg}(1 \mathrm{mmol}) \mathbf{1 a}, 153 \mathrm{mg}(1.09 \mathrm{mmol}) \mathbf{2 b}$ and $136 \mathrm{mg}$ (1.25 mmol) 3c were reacted with $76 \mathrm{mg}$ montmorillonite in $2 \mathrm{~mL}$ toluene. After work up and column chromatography $\left(\mathrm{CH}_{2} \mathrm{Cl}_{2} / \mathrm{TBME}=15 / 1\right) 320 \mathrm{mg}(0.98 \mathrm{mmol}) \mathbf{4 j}(98 \%)$ were isolated as a pale gray solid.

mp 191-192 ${ }^{\circ} \mathrm{C}\left(\right.$ Lit. $\left.^{10 \mathrm{a}} \mathrm{mp} 179-181^{\circ} \mathrm{C}\right) .{ }^{1} \mathrm{H}-\mathrm{NMR}\left(300 \mathrm{MHz}, \mathrm{CDCl}_{3}\right): \delta=1.19(3 \mathrm{H}$, overlapped, 4' '-Ha, 3' '-Ha, 5''-Ha), 1.29 (2H, bt, $J=11.5 \mathrm{~Hz}, 2$ ''-Ha, 6'-Ha), 1.61 (1H, bs, 4' '-Hb), 1.72 (2H, m, 3' '-Hb, 5' -Hb), 1.83 (2H, m, 2''-Hb, 6'-Hb), 2.93- 2.95 (1H, m, 1' '-H), 3.05 (1H, d, J $=4.5 \mathrm{~Hz}, \mathrm{NH}), 6.79(1 \mathrm{H}, \mathrm{ddd}, J=1.1 \mathrm{~Hz}, J=6.8 \mathrm{~Hz}, J=6.8 \mathrm{~Hz}, 5-\mathrm{H}), 7.14(1 \mathrm{H}, \mathrm{ddd}, J=1.2$ $\mathrm{Hz}, J=6.8 \mathrm{~Hz}, J=8.9 \mathrm{~Hz}, 6-\mathrm{H}), 7.40\left(2 \mathrm{H}, \mathrm{m}, 3^{\prime}-\mathrm{H}, 5^{\prime}-\mathrm{H}\right), 7.53(1 \mathrm{H}, \mathrm{dt}, J=0.9 \mathrm{~Hz}, J=9.0 \mathrm{~Hz}$, 7-H), 8.02 (2H, m, 2'-H, 6'-H), 8.07 (1H, dt, $J=1.1 \mathrm{~Hz}, J=6.8 \mathrm{~Hz}, 4-\mathrm{H}) .{ }^{13} \mathrm{C}-\mathrm{NMR}(75 \mathrm{MHz}$, $\mathrm{CDCl}_{3}$ ): $\delta=24.77$ (3'’-C, 5' '-C), 25.65 (4' '-C), 34.17 (2'”-C, 6' '-C), 56.82 (1'”-C), 111.98 (5C), 117.12 (7-C), 122.74 (4-C), 124.61 (6-C), 124.91 (3-C), 128.25 (2'-C, 6'-C), 128.65 (3'-C, 5'-C), 132.44 (1'-C), 133.13 (2-C), 135.06 (4'-C), 141.29 (7a-C). MS (70 eV, EI): $m / z(\%)=325$ (62) $\left[\mathrm{M}^{+}\right], 242$ (100), 215 (58), 78 (25). HRMS (70 eV, EI) for $\mathrm{C}_{19} \mathrm{H}_{20} \mathrm{ClN}_{3}$ : Calcd.: 325.1300; Found: 325.1354 .

\section{Acknowledgements}

We thank Dr. A. Job, Lanxess for guanidinium salts and Ms. S. Mika for recording NMR spectra. Financial support by the BMBF (01R105181) is greatly acknowledged.

\section{References}

1. Loupy, A. Ed. Microwaves in Organic Synthesis, 2nd Edn.; Wiley-VCH: Weinheim, 2006. (b) Kappe, C. O.; Stadler, A. Microwaves in Organic and Medicinal Chemistry; Wiley-VCH: Weinheim, 2005.

2. Wasserscheid, P.; Welton, T. Eds., Ionic Liquids in Synthesis, 2nd Edn.; Wiley-VCH: Weinheim, 2007.

3. Isambert, N.; Sanchez Duque, M. M; Plaquevent, J.-C.; Génisson, Y.; Rodriguez, J.; Constantieux, T. Chem. Soc. Rev. 2011, 40, 1347. (b) Martins, M. A. P.; Frizzo, C. P.; Moreira, D. N.; Zanatta, N.; Bonacorso, H. G. Chem. Rev. 2008, 108, 2015. (c) El Ashry, E. S. H.; Kassem, A. A.; Ramadan, E. in Advances in Heterocyclic Chemistry; Katritzky, A. R. Ed.; Elsevier: Amsterdam, 2006; Vol. 90, p. 1. (d) El Ashry, E. S. H.; Ramadan, E.; Kassem, A. A.; Hagar, M. In Advances in Heterocyclic Chemistry; Katritzky, A. R. Ed.; Elsevier: Amsterdam, 2005; Vol. 88, p. 1

4. Giernoth, R. Angew. Chem. Int. Ed. 2010, 49, 2834. (b) Domínguez de María, P. Angew. Chem. Int. Ed. 2008, 47, 6960. (c) Bica, K. Gaertner, P. Eur. J. Org. Chem. 2008, 3235. 
5. Polshettiwar, V.; Varma, R. S. Acc. Chem. Res. 2008, 41, 629. (b) Habermann, J.; Ponzi, S.; Ley, S. V. Mini-Rev. Org. Chem. 2005, 2, 125. (c) Leadbeater, N. E.; Torenius, H. M.; Tye, H. Comb. Chem. High Throughput Screening 2004, 7, 511.

6. Shah, J.; Blumenthal, H.; Yacob, Z.; Liebscher, J. Adv. Synth. Catal. 2008, 350, 1267. (b) Li, S.; Lin, Y.; Xie, H.; Zhang, S.; Xu, J. Org. Lett. 2006, 8, 391. (c) Zhu, A.; Jiang, T.; Han, B.; Huang, J.; Zhang, J.; Ma, X. New J. Chem. 2006, 30, 736. (d) Branco, L. C. P.; Gois, P. M. P.; Lourenço, N. M. T.; Kurteva, V. B.; Afonso, C. A. M. Chem. Commun. 2006, 2371. (e) Xie, H.; Zhang, S.; Duan, H. Tetrahedron Lett. 2004, 45, 2013.

7. Fang, S.; Yang, L.; Wang, J.; Li, M.; Tachibana, K.; Kamijima, K. Electrochimica Acta 2009, 54, 4269. (b) Fang, S.; Yang, L.; Wang, J.; Zhang, H.; Tachibana, K.; Kamijima, K. J. Power Sources 2009, 191, 619. (c) Gao, Y.; Arritt, S. W.; Twamley, B.; Shreeve, J. M. Inorg. Chem. 2005, 44, 1704. (d) Wang, P.; Zakeeruddin, S. M.; Grätzel, M.; Kantlehner, W.; Mezger, J.; Stoyanov, E. V.; Scherr, O. Appl. Phys. A 2004, 79, 73. (e) Mateus, N. M. M.; Branco, L. C.; Lourenço, N. M. T.; Afonso, C. A. M. Green Chemistry 2003, 5, 347.

8. Orru, R. V. A.; Ruijter, E. (Eds.) Synthesis of Heterocycles via Multicomponent Reactions; Springer: Heidelberg, 2010; Vol. 1 and 2. (b) Touré, B. B.; Hall, D. G. Chem. Rev. 2009, 109, 4439. (c) Zhu, J.; Bienaymé, H. (Eds.) Multicomponent Reactions; Wiley-VCH: Weinheim, 2005. (d) Ramón, D. J.; Yus, M. Angew. Chem. Int. Ed. 2005, 44, 1602.

9. Katritzky, A. R.; Xu, Y.-J.; Tu, H. J. Org. Chem. 2003, 68, 4935. (b) Abe, Y.; Kayakiri, H.; Satoh, S.; Inoue, T.; Sawada, Y.; Imai, K.; Inamura, N.; Asano, M.; Hatori, C.; Katayama, A.; Oku, T.; Tanaka H. J. Med. Chem. 1998, 41, 564. (c) Gueiffier, A.; Mavel, S.; Lhassani, M.; Elhakmaoui, A.; Snoeck, R.; Andrei, G.; Chavignon, O.; Teulade, J.-C.; Witvrouw, M.; Balzarini, J.; De Clercq, E.; Chapat, J.-P. J. Med. Chem. 1998, 41, 5108. (d) Gueiffier, A.; Lhassani, M.; Elhakmaoui, A.; Snoeck, R.; Andrei, G.; Chavignon, O.; Teulade, J.-C.; Kerbal, A.; Essassi, E. M.; Debouzy, J.-C.; Witvrouw, M.; Blache, Y.; Balzarini, J.; De Clercq, E.; Chapat, J.-P. J. Med. Chem. 1996, 39, 2856. (e) Elhakmaoui, A.; Gueiffier, A.; Milhavet, J.-C.; Blache, Y.; Chapat, J.-P.; Chavignon, O.; Teulade, J.-C.; Snoeck, R.; Andrei, G.; De Clercq, E. Bioorg. Med. Chem. Lett. 1994, 4, 1937. (f) Sanflippo, P. J.; Urbanski, M.; Press, J. B.; Dubinsky, B.; Moore, J. B., Jr. J. Med. Chem. 1988, 31, 2221.

10. Adib, M.; Mahdavi, M.; Noghani, M. A.; Mirzaei, P. Tetrahedron Lett. 2007, 48, 7263. (b) Rousseau, A. L.; Matlaba, P.; Parkinson, C. J. Tetrahedron Lett. 2007, 48, 4079. (c) Shaabani, A.; Soleimani, E.; Maleki, A. Tetrahedron Lett. 2006, 47, 3031. (d) Ireland, S. M.; Tye, H.; Whittacker, M. Tetrahedron Lett. 2003, 44, 4369. (e) Blackburn, C.; Guan, B. Tetrahedron Lett. 2000, 41, 1495. (f) Varma, R. S.; Kumar, D. Tetrahedron Lett. 1999, 40, 7665. (g) Blackburn, C. Tetrahedron Lett. 1998, 39, 5469. (h) Bienaymé, H.; Bouzid, K. Angew. Chem. Int. Ed. 1998, 37, 2234. (i) Groebke, K.; Weber, L.; Mehlin, F. Synlett 1998, 661. 